\title{
Urine My Heart: A Case of Aerococcal Endocarditis
}

\author{
Marium Khan ${ }^{1}$, Harpreet Gill ${ }^{1}$, Mudassir Khan ${ }^{2}$, Vishmayaa Saravanan ${ }^{1}$, Pinky Jha ${ }^{1}$ \\ 1. Internal Medicine, Medical College of Wisconsin, Milwaukee, USA 2. Revenue Cycle, Providence St. Joseph Health, \\ Tegria Consulting, Renton, USA
}

Corresponding author: Marium Khan, markhan@mcw.edu

\begin{abstract}
An elderly woman with previously known valvular disease presented to the emergency department due to altered mental status. In addition to obtaining an infectious work-up, a bedside echocardiogram was performed and revealed right heart strain prompting a formal echocardiogram evaluation and treatment for a possible pulmonary embolism. Initial laboratory work returned with blood and urine cultures positive for Aerococcus urinae. A transthoracic echocardiogram further revealed new aortic regurgitation. Given this, a transesophageal echocardiogram was completed, confirming new aortic insufficiency as well as findings of infective endocarditis. She did not undergo surgical intervention; however, she was discharged with a plan to continue intravenous antibiotics for six weeks. Although typically seen in genitourinary infections, $A$. urinae is a rare cause of infective endocarditis and is increasingly identified due to improved speciation techniques. We describe a unique presentation of invasive $A$. urinae infection to increase awareness and further research on a less commonly encountered bacteria that may present as a urinary tract infection and has the potential to cause invasive disease.
\end{abstract}

Categories: Cardiology, Internal Medicine, Infectious Disease

Keywords: aerococcus urinae, endocarditis, urinary tract infection, mass spectrometry

\section{Introduction}

Aerococcus urinae is a rare gram-positive coccus seen in cystitis or urinary tract infections (UTI). Risk factors include older age, male sex, and pre-existing urinary tract abnormalities or benign prostatic hyperplasia (BPH) [1]. While aerococcal infections may be considered low in pathogenicity, they may result in invasive infections including bacteremia, endocarditis, peritonitis, meningitis, osteomyelitis, joint infections, or spondylodiscitis [2]. Historically, aerococcus was misidentified due to similarities with other gram-positive cocci; however, more infections are becoming properly identified due to improved mass spectrometry techniques. We describe a unique case of aerococcal endocarditis in an elderly woman with previously known valvular disease.

Review began 04/07/2021 Review ended 04/16/2021 Published 04/20/2021

\section{○ Copyright 2021} Khan et al. This is an open access article distributed under the terms of the Creative Commons Attribution License CC-BY 4.0., which permits unrestricted use, distribution, and reproduction in any medium, provided the original author and source are credited.

\section{Case Presentation}

An 86-year-old woman with a history of atrial fibrillation, aortic stenosis, and hypertension presented to the emergency department due to altered mental status. She was lethargic, confused, and was not answering questions appropriately for the past four days. On arrival, she was afebrile, and her vitals included a pulse of 139 beats per minute, blood pressure of $110 / 79 \mathrm{mmHg}$, and a respiratory rate of 16 breaths per minute with saturation of $96 \%$ on room air. Physical exam was significant for a slow to respond female, orientated to self, who was able to follow commands with no focal neurological deficit. Her skin was warm and well-perfused with normal capillary refill, with no rashes or petechiae. The cardiovascular exam was significant for tachycardia, an irregular heart rhythm, and a systolic murmur heard best at the right upper sternal border. She had lower extremity pitting edema bilaterally. Her lab results were notable for elevated white blood cell count (WBC) $25.2 \times 10^{3} \mu \mathrm{L}\left(3.9-11.3 \times 10^{3} \mu \mathrm{L}\right)$, hemoglobin $11.0 \mathrm{~g} / \mathrm{dL}(11.3-15.1 \mathrm{~g} / \mathrm{dL})$, platelets $58.0 \times 10^{3} \mu \mathrm{L}$ $\left(165-366 \times 10^{3} \mu \mathrm{L}\right)$, troponin $0.164 \mathrm{ng} / \mathrm{mL}(<0.010 \mathrm{ng} / \mathrm{mL})$, lactic acid $2.5 \mathrm{mmol} / \mathrm{L}(0.5-2.0 \mathrm{mmol} / \mathrm{L})$, and a basic chemistry panel was within normal limits. The urinalysis was remarkable for the presence of WBCs, leukocyte esterase, and bacteria with a urine culture pending. Additionally, two sets of blood cultures were obtained. Her electrocardiogram was significant for atrial fibrillation with a rapid ventricular rate (RVR). She was started on ceftriaxone for probable UTI and was admitted to the hospital for further management. Further diagnostics included lower extremity Doppler ultrasound, which revealed deep venous thrombosis (DVT). A bedside echocardiogram indicated right ventricular strain, and the N-terminal proB-type natriuretic peptide level was elevated at $8,215 \mathrm{pg} / \mathrm{mL}(<449 \mathrm{pg} / \mathrm{mL})$. Due to concern for possible pulmonary embolism in the setting of known DVT, the patient was started on a continuous heparin infusion. A transthoracic echocardiogram revealed severe aortic valve calcification with new aortic insufficiency. Blood and urine cultures returned positive for Aerococcus urinae. A transesophageal echocardiogram further confirmed moderate to severe aortic regurgitation with findings suggestive of infective endocarditis (IE), see Figures 1,2. 


\section{Cureus}

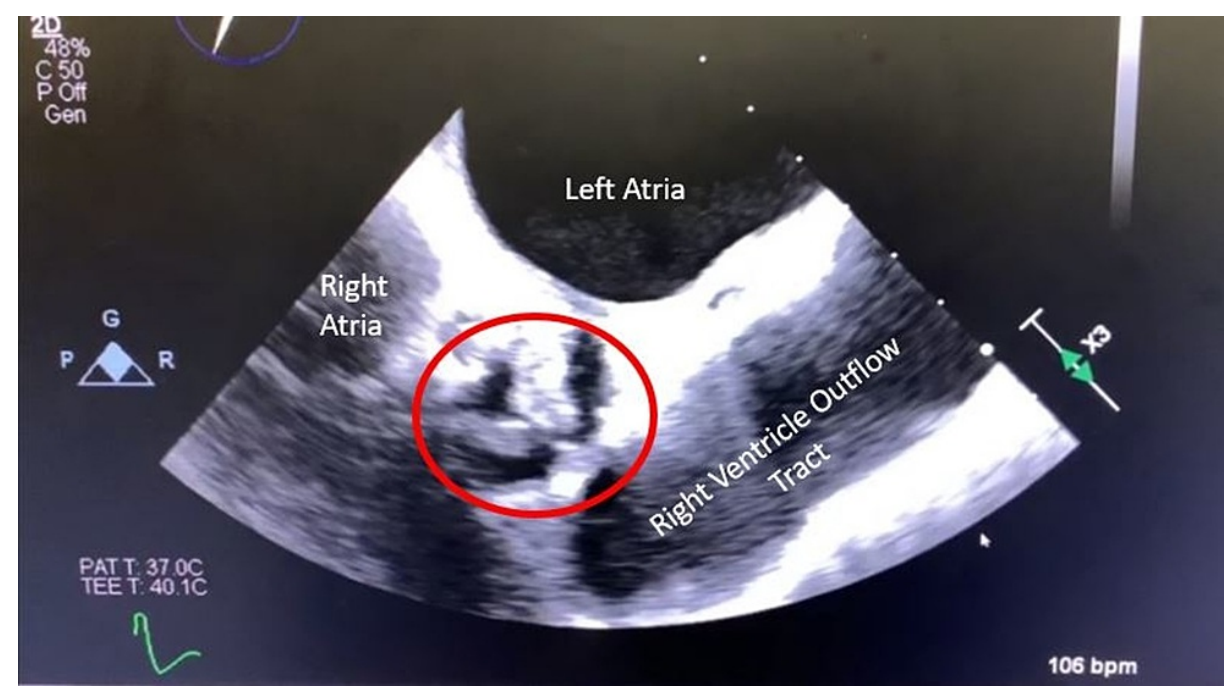

FIGURE 1: Transesophageal echocardiogram short-axis view with aortic valve vegetation circumscribed

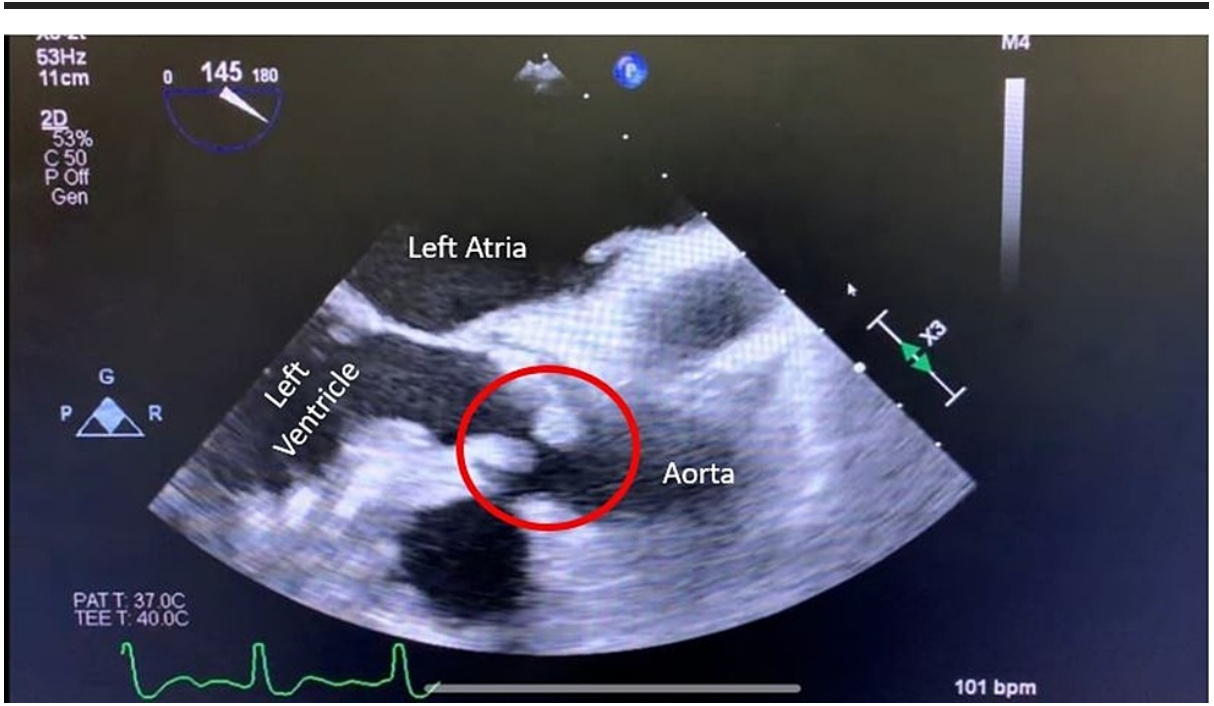

FIGURE 2: Transesophageal echocardiogram three-chamber view with aortic valve vegetation circumscribed

Antibiotics were expanded to include gentamicin with ceftriaxone for IE, and surgical evaluation was consulted. Given she was a high-risk surgical candidate, surgery intervention was not offered at this time unless evidence of further clinical decompensation. Additional surgical recommendations included reevaluation after completion of antibiotic course. She was therefore discharged on intravenous antibiotics to complete a total of six weeks of treatment with ceftriaxone and two weeks of gentamicin. Soon after completion, the patient returned due to worsening thrombocytopenia that had previously improved upon discharge. On readmission, repeat labs were most notable for worsening thrombocytopenia, leukocytosis, acute kidney injury, and hyperkalemia. The patient was in atrial fibrillation with RVR and physical exam concerning for decompensated heart failure. Despite administration of broad-spectrum antibiotics and heart rate control, the patient continued to decline, and her clinical picture was consistent with cardiogenic shock. In addition to respiratory distress, the patient went into ventricular fibrillation arrest. Due to the patient's wishes to not be intubated or resuscitated, she was made comfortable and passed away.

\section{Discussion}

The aerococci family of bacteria was first identified in 1953 and has become increasingly implicated in human disease [3]. In 1992, A. urinae was discovered by Aguirres and Collins in urine samples as a rare causative agent in UTIs [4]. Additional species within the aerococal genus include A. viridans, A. christensenii, A. sanguinicola, and A. urinaehominis [5,6]. A. urinae is an alpha-hemolytic, catalase-negative, gram-positive cocci that can appear as diplococci, tetrads, and even clusters [5]. This diverse presentation and similarity to 
more commonly pathologic bacteria such as Staphylococci, Streptococci, and Enterococci make A. urinae difficult to identify by culture and likely underdiagnosed.

These qualities, in addition to some commercial labs not including tests specific for $A$. urinae, complicate making the diagnosis. In their review of $63 \mathrm{~A}$. urinae UTI cases, Zhang et al. found that while the gold standard diagnostic test was sequencing of the $16 \mathrm{~S}$ ribosomal RNA (rRNA), biochemical detection of leucine arylamidase, beta-glucuronidase, and pyrrolidonyl arylamidase (PYR) was an effective and more routine option [5]. In recent years, mass spectroscopy has become a powerful tool for identifying microbial organisms. Matrix-assisted laser desorption ionization-time of flight mass spectrometry (MALDI-TOF MS) in particular is well-documented in identifying A. urinae using non-culture techniques [1]. Conventional methods of UTI identification require 18-48 hours of culturing, while MALDI-TOF MS reduces processing time to under an hour with excellent specificity and sensitivity, further minimizing inappropriate empirical antimicrobial therapy before identification [7].

Other commercially available methods are unable to safely separate aerococci from other bacteria or differentiate aerococcal species from one another [2]. Senneby’s study of MALDI-TOF MS concluded a 94\% median probability of correct species identification of $A$. urinae isolates while attaining a pooled specificity of $93 \%$, which is in accordance with strong identification capabilities [2].

In addition to UTIs, $A$. urinae has been linked in elderly populations with comorbidities to more invasive infections [8]. In rare instances, the bacteria exit the urogenital tract hematogenously and can cause more severe, systemic complications. These presentations include necrotizing soft tissue infections, spondylodiscitis, perineal abscesses, lymphadenitis, and meningitis $[1,8,9]$. In a majority of cases, underlying UTI is suspected and typically has a favorable prognosis [8]. Although invasive disease is possible, literature documenting these cases is limited. For example, at the time of writing, PubMed research yielded less than 10 case reports of spondylodiscitis presentation with $A$. urinae.

IE is another systemic complication of $A$. urinae infection, as seen in the case above. In the bloodstream, $A$. urinae can seed within the endocardium in areas of disruption due to valvular disease, foreign bodies such as pacemakers, disrupted blood flow, or calcifications. In the United States, there are approximately 40-50,000 cases annually of IE [10]. Risk factors such as intravenous drug use (IVDU), intravascular catheters, immunosuppression, degenerative heart disease, diabetes, cancer, and age have largely replaced rheumatic heart disease as a cause of IE in developed countries [10,11].

S. aureus, a gram-positive aerobic organism, is the most recognized causative agent of IE, representing almost one-third of cases [10]. Conversely, the prevalence of $A$. urinae in IE is thought to be three in $1,000,000$ [12]. While the method behind which $A$. urinae causes IE remains to be elucidated, scientific literature proposes an underlying mechanism of cellular aggregation.

Like $S$. aureus, A. urinae has a strong propensity to form biofilms, which is an important step in its pathophysiology. A. urinae is able to produce a protective biofilm to induce platelet aggregations and ultimately form the vegetation within the endocardium [13]. A recent study by Yaban et al. used fluorescence in situ hybridization (FISH)-probe analysis to confirm two cases of in-vivo biofilm production by A. urinae [14]. Biofilm production by $A$. urinae allows for the bacteria to settle not only within the urinary tract system but as well as within the endocardium, including heart valves. Once the bacteria are able to adhere onto these surfaces, it aggregates along with platelets and other inflammatory response cells increasing the risk of vegetation formation [6].

A. urinae incidence increases with age and tends to present more in men than women [1,5,13]. A 2018 review by Yabes et al. found only 43 cases in the literature with a mean age of 73 and in which over $80 \%$ were men (n = 36) [1]. Presence of comorbid urogenital abnormalities is associated with a higher risk of A. urinae infection. Other risk factors include frequent UTIs, prostatitis, BPH, or an indwelling Foley catheter [1]. The increased prevalence in males may be linked to development of prostatic hyperplasia and incidence of bacterial prostatitis $[15,16]$. Although Enterobacteriaceae are the most common causative organisms in both acute bacterial prostatitis (ABP) and chronic bacterial prostatitis (CBP), gram-positive organisms are found in both types and more commonly in CBP [16]. Despite the increased risk of A. urinae infection among males and those with pre-existing urogenital abnormalities, our case patient presented without the typical urogenital risk factors. However, she did have previously known valvular disease and was 86 years old, likely predisposing her to IE.

While there are no specific recommendations, $A$. urinae is sensitive to beta-lactam medications and resistant to sulfonamides and most notably fluoroquinolones, which are commonly used to treat bacterial prostatitis $[1,16,17]$. In particular, A. urinae IE responds well to beta-lactams and aminoglycosides. In all 43 cases reviewed by Yabes et al., this was the mainstay of the treatment [1]. Vancomycin therapy can be considered for more severe cases or individuals allergic to penicillin [1].

Synergistic behavior with beta-lactams and aminoglycosides can last anywhere from 10 days to six weeks [1]. Despite the empirical success of beta-lactams and aminoglycosides for treatment options, the approach is 
not universal. One of the greatest variables limiting prolonged use of aminoglycosides includes toxicity such as nephrotoxicity and ototoxicity [18]. Furthermore, in one study, A. urinae isolates failed to show synergistic effects of beta-lactam gentamicin therapy approximately $50 \%$ of the time [19]. Given that possible outcomes range from synergistic effects to drug resistance and fatal consequences, timely and accurate identification of $A$. urinae using improved techniques is of vital importance in appropriate treatment strategy. In addition to antibiotic therapy, evaluation for removal of the vegetation should be considered by expert cardiothoracic surgery consultation.

\section{Conclusions}

Although presentation of $A$. urinae is uncommon, clinicians should be aware of the possibility of invasive $A$. urinae infections such as IE as seen in our case patient. Due to improved diagnostic techniques in microbiology identification, more cases of $A$. urinae are being identified allowing for prompt and proper treatment. This case report adds to the limited literature on invasive $A$. urinae infections, including IE. Further research must be conducted in order to develop guidelines on treatment and management of $A$. urinae infections. As clinicians, we must continue to have a high index of suspicion for more invasive disease when identifying $A$. urinae infections.

\section{Additional Information \\ Disclosures}

Human subjects: Consent was obtained or waived by all participants in this study. Conflicts of interest: In compliance with the ICMJE uniform disclosure form, all authors declare the following: Payment/services info: All authors have declared that no financial support was received from any organization for the submitted work. Financial relationships: All authors have declared that they have no financial relationships at present or within the previous three years with any organizations that might have an interest in the submitted work. Other relationships: All authors have declared that there are no other relationships or activities that could appear to have influenced the submitted work.

\section{Acknowledgements}

We thank Dr. Mrigank Gupta for his contribution in editing of this manuscript.

\section{References}

1. Yabes JM, Perdikis S, Graham DB, Markelz A: A rare case of Aerococcus urinae infective endocarditis in an atypically young male: case report and review of the literature. BMC Infect Dis. 2018, 18:522. 10.1186/s12879-018-3414-0

2. Senneby E, Nilson B, Petersson AC, Rasmussen M: Matrix-assisted laser desorption ionization-time of flight mass spectrometry is a sensitive and specific method for identification of aerococci. J Clin Microbiol. 2013, 51:1303-4. 10.1128/JCM.02637-12

3. Williams RE, Hirch A, Cowan ST: Aerococcus, a new bacterial genus . J Gen Microbiol. 1953, 8:475-80. 10.1099/00221287-8-3-475

4. Aguirre M, Collins MD: Phylogenetic analysis of some Aerococcus-like organisms from urinary tract infections: description of Aerococcus urinae sp. nov. J Gen Microbiol. 1992, 138:401-5. 10.1099/00221287138-2-401

5. Zhang Q, Kwoh C, Attorri S, Clarridge JE 3rd: Aerococcus urinae in urinary tract infections . J Clin Microbiol. 2000, 38:1703-5. 10.1128/JCM.38.4.1703-1705.2000

6. Rasmussen M: Aerococci and aerococcal infections. J Infect. 2013, 66:467-74. 10.1016/j.jinf.2012.12.006

7. Tang M, Yang J, Li Y, Zhang L, Peng Y, Chen W, Liu J: Diagnostic accuracy of MALDI-TOF mass spectrometry for the direct identification of clinical pathogens from urine. Open Med (Wars). 2020, 15:26673. 10.1515/med-2020-0038

8. Lyagoubi A, Souffi C, Baroiller V, Vallee E: Aerococcus urinae spondylodiscitis: an increasingly described localization. EJIFCC. 2020, 31:169-73.

9. Forsvall A, Wagenius M, Rasmussen M: Perigenital necrotizing soft tissue infection caused by Aerococcus urinae. IDCases. 2019, 18:e00590. 10.1016/j.idcr.2019.e00590

10. Cahill TJ, Prendergast BD: Infective endocarditis. Lancet. 2016, 387:882-93. 10.1016/S0140-6736(15)000677

11. Holland TL, Baddour LM, Bayer AS, Hoen B, Miro JM, Fowler VG Jr: Infective endocarditis. Nat Rev Dis Primers. 2016, 2:16059. 10.1038/nrdp.2016.59

12. de Jong MF, Soetekouw R, ten Kate RW, Veenendaal D: Aerococcus urinae: severe and fatal bloodstream infections and endocarditis. J Clin Microbiol. 2010, 48:3445-7. 10.1128/JCM.00835-10

13. Ludhwani D, Li J, Huang EE, Sikora A, Thomas G: Aerococcus urinae aortic valve endocarditis with kissing aortic wall ulcer: a case report and literature review. Am J Case Rep. 2020, $21: e 920974$. 10.12659/AJCR.920974

14. Yaban B, Kikhney J, Musci M, et al.: Aerococcus urinae - a potent biofilm builder in endocarditis . PLoS One. 2020, 15:e0231827. 10.1371/journal.pone.0231827

15. Tathireddy H, Settypalli S, Farrell JJ: A rare case of aerococcus urinae infective endocarditis . J Community Hosp Intern Med Perspect. 2017, 7:126-9. 10.1080/20009666.2017.1314072

16. Davis NG, Silberman M: Bacterial Acute Prostatitis. StatPearls Publishing, Treasure Island, FL; 2020.

17. Rasmussen M: Aerococcus: an increasingly acknowledged human pathogen. Clin Microbiol Infect. 2016, 22:22-7. 10.1016/j.cmi.2015.09.026 


\section{Cureus}

18. Gonzalez LS 3rd, Spencer JP: Aminoglycosides: a practical review. Am Fam Physician. 1998, 58:1811-20.

19. Sunnerhagen T, Nilson B, Olaison L, Rasmussen M: Clinical and microbiological features of infective endocarditis caused by aerococci. Infection. 2016, 44:167-73. 10.1007/s15010-015-0812-8 